\title{
Teratoma of the Neck on Fine Needle Aspiration Cytology: An Unusual Case Presentation
}

\author{
Challa Sukumar ${ }^{1}$, Aarzoo Jahan ${ }^{1 *}$, Shakti Kumar Yadav², Sompal Singh ${ }^{1}$ and Namrata Sarin ${ }^{1}$ \\ 'Department of Pathology North Delhi Municipal Corporation Medical College and Hindu Rao Hospital, Delhi, India \\ ${ }^{2}$ Department of Pathology, All India Institute of Medical Sciences, New Delhi
}

\begin{abstract}
Teratomas are tumours which are derived from all the 3 germ cell layers: endoderm, mesoderm and ectoderm with varying proportions. Cervical teratomas in children are almost always benign but locally aggressive. They can present with respiratory distress and immediate excision is required.The cervical teratoma is an uncommon entity which are rarely reported in Indian medical literature particularly on FNA. We present a case of ananterior cervical teratoma in an infant which was diagnosed on the base of FNA material.
\end{abstract}

Keywords: Teratomas, Germ Cell Layer, Cervical Teratomas, Sacrococcygeal Region, Chondrodystrophia Fetalis.

\section{Introduction}

The term teratoma is derived from a Greek word which means monster. Teratomas are germ cell tumours (neoplasm) which can have their origin from all the three germ cell layers namely ectoderm, mesoderm, and endoderm. Location-wise, these tumours have ubiquitous distribution in body with various frequency site distribution. Most commonly teratomas occur in the testes or ovaries (gonads) or the lower back (sacrococcygeal region). These tumours are extremely rare in neck region consisting of $3 \%$ of all teratomas. ${ }^{[1]}$ The sign and symptoms are due to the mass effect of these lesions, making them potentially lethal. The specific symptoms associated with cervical teratomas in children vary depending upon the size of the tumor. Small tumors may not cause any symptoms (asymptomatic). However, a large teratoma may cause disfigurement and compress nearby structures such as the windpipe (trachea). Such large tumors may cause additional symptoms including a wheezing or whistling sound when breathing (stridor), difficulty breathing, or shortness of breath (dyspnea). These tumors can also compress the esophagus, inhibiting swallowing. Eventually, compression of vital structures may cause life-threatening complications such as respiratory distress. ${ }^{[2]}$ Unlike adults, cervical teratomas in children are often congenital and very rarely associated with malignancy. They are almost always benign but locally aggressive.

\section{Case Report}

A 08-months-old female infant was presented to Hindu Rao Hospital, Delhi with patient presented with a large midline anterior cervical mass since birth. The patient remained asymptomatic and the patient's parents were worried about the large mass in neck for which they had approached the hospital. There is swelling in anterior cervical region since birth and gradually increasing in size. There is no complaint of dyspnea or pain.

On physical examination, there was a diffuse, nodular mid line neck swelling which was irregular of size about $8 \times 8 \mathrm{~cm}$ having firm consistency with moderate mobility without tenderness and no attachment to overlying skin was noted.

USG showed that both bilateral thyroid gland and submandibular gland was normal and involved by neck mass. A large mass of size $9.5 * 7 \mathrm{~cm}$ in the anterior cervical region was noted with a low out flow suggestive of lymphovascular lesion. MRI and CT scan reports were not available.

Fine needle aspiration cytology was performed twice from anterior cervical region and the smears were made. Cellular smears examined showed heterogenous population of cells comprising of predominately mature nucleated squamous epithelial cell, some mature fibroadipose tissue fragments, columnar to spindle cells, clusters of cells with abundant vacuolated cytoplasm along with associated scant eosinophilic stromal material and some sheets of benign looking epithelial cells with glandular differentiation noted in hemorrhagic background.

Apart cell block was prepared from the FNA material and the corresponding $\mathrm{H}$ \& $\mathrm{E}$ slides were examined which showed mature cartilage, mature fibroadipose tissue fragments, some keratinous material and neuroglial tissue.

Immunohistochemistry was doing using glial fibrillary acid protein (GFAP) antibody which show cytoplasmic 
positivity. Based on all these findings a final diagnosis of teratoma, anterior cervical region was made and advised for complete excision for urgent histopathological evaluation was made.

\section{Discussion}

The word teratoma is derived from Greek, which means monstrous. Teratoma are found everywhere in the body, commonest location being gonads or sacral region. These tumours are extremely rare in cervical region which constitutes a small percentage of $1.6 \%$ to $9.3 \%$ of all pediatric teratomas, roughly equating to 1 per 40,000 births. They predominately occur in females $(3 / 4$ of the cases). ${ }^{[3]}$

The aetiology of cervical teratomas is still confused. These tumors seem to develop randomly for no apparent reason (sporadic). Research is going on to learn about the probable causative factors. The studies have centered around the genetic mutations leading to amplification, 9 p22

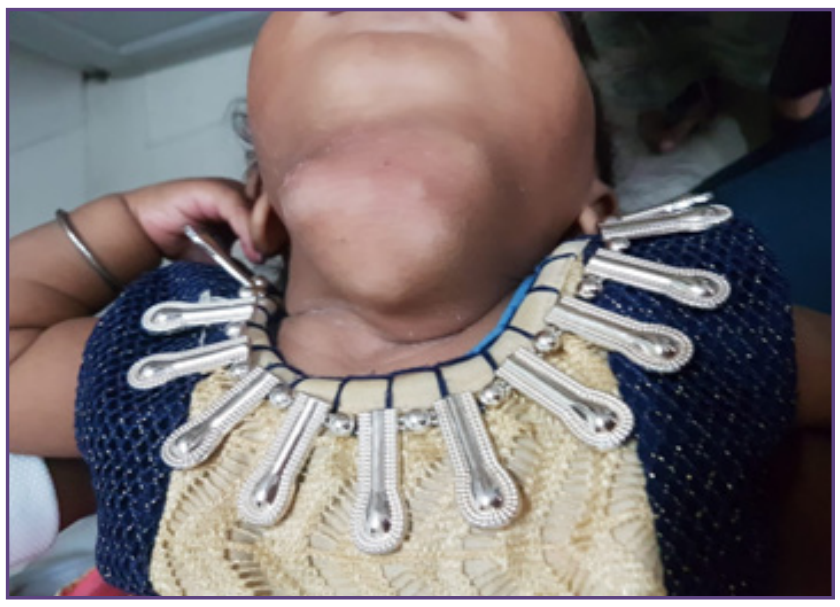

Fig. 1: Patient with anterior cervical teratoma.

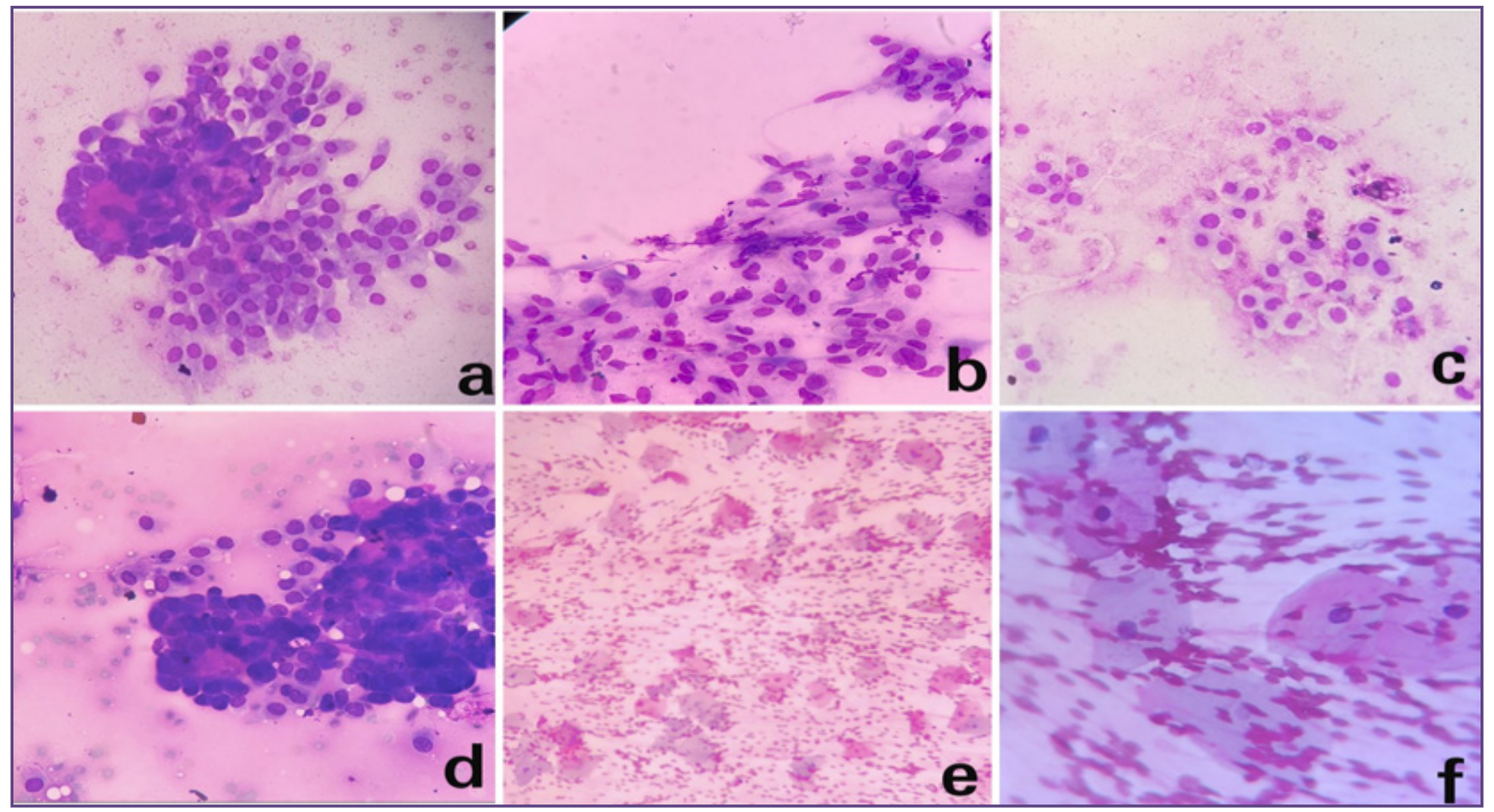

Fig. 2: Columnar cells \& stomal material (a, 400x, MGG), spindle cells (b, 400x, MGG), vacuolated cytoplasm, clear cells(c, 400x, MGG), glandular differentiation (d, 400x, MGG), squamous cells (100x, Pap stain), squamous cells (400x, Pap stain). 


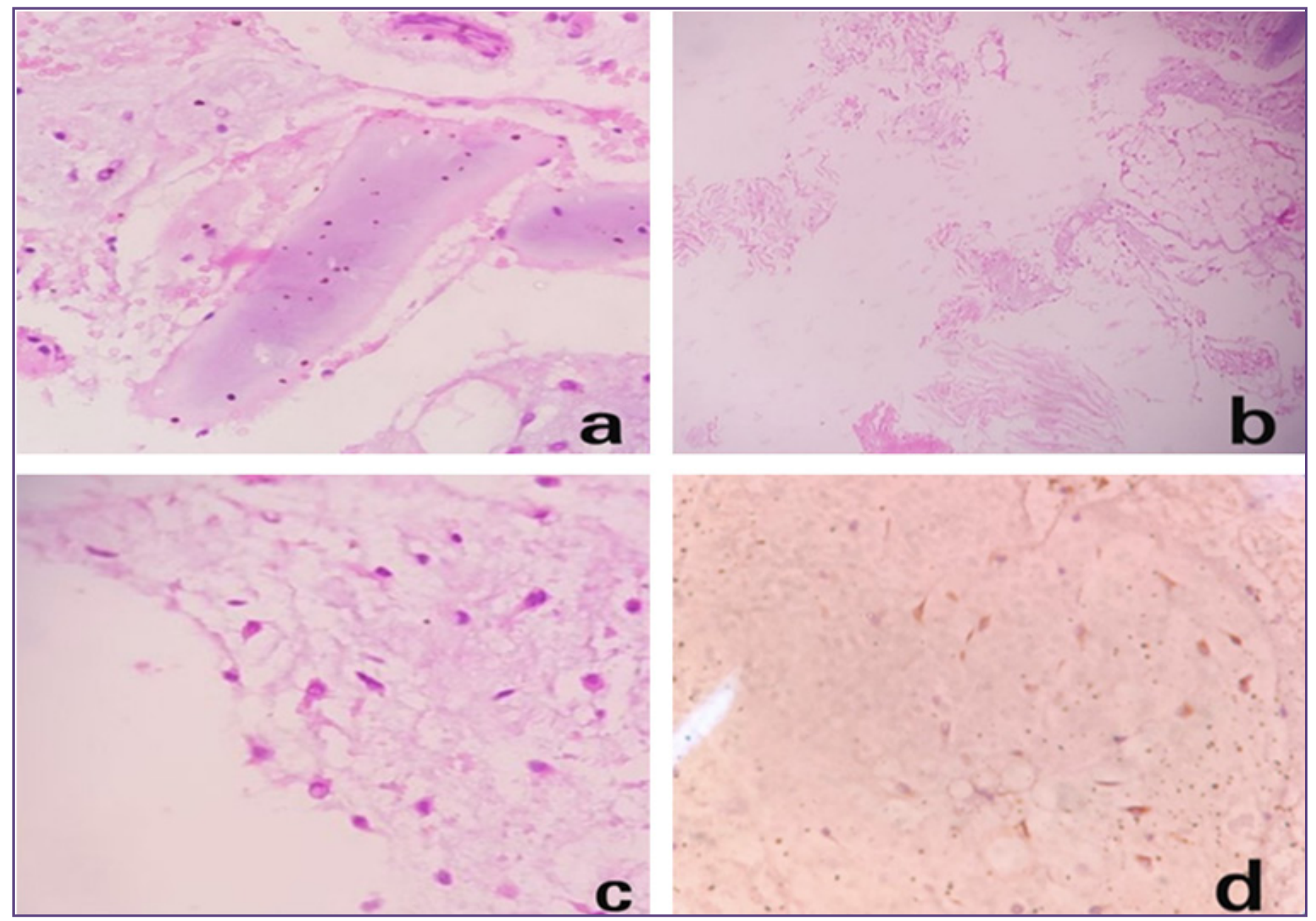

Fig. 3: Cartilage $(a, 400 x, H \& E)$, keratin material and adipose tissue (b, 400x, H\&E), neurological component (400x, H\&E), GFAP positive $(400 x, D A B)$.

deletion, and 17q21.33 1-copy gain. ${ }^{[4]}$ The malformations associated with teratoma include imperforate anus, Chondrodystrophia fetalis, hypoplastic left ventricle with pulmonary hypoplasia, cystic fibrosis, absence of corpus callosum, and arachnoid cyst, though very rare.

Symptoms associated with cervical teratomas depends upon the size of the tumor. Small tumors may not cause any symptoms (asymptomatic). However large teratoma may cause disfigurement and compress nearby structures such as the trachea and causes a wheezing or whistling sound when breathing (stridor), difficulty breathing, or shortness of breath (dyspnea). These tumors can also compress the esophagus and inhibit swallowing. Eventually, compression of vital structures may cause life-threatening complications such as respiratory distress. If the mass is found after birth, ultrasonography, computed tomography (CT) and MRI are of primary importance for determining the extension, involvement of adjacent structures, and helps in planning surgery. ${ }^{[5]}$
Prenatal diagnosis of cervical teratomas is possible via ultrasound. Large tumors may be readily apparent on a fetal ultrasound, the neck may appear hyper-extended, or associated findings such as excessive amniotic fluid (polyhydramnios) may be detected. In addition to ultrasound, additional specialized imaging techniques such as computerized tomography (CT) scanning and magnetic resonance imaging (MRI) may be used to evaluate the size, placement, and extension of a tumor for future surgical procedures.

Degree of malignancy depends upon the grade of maturity of the component tissues. Most of the cervical teratomas occurring in children are non-cancerous (benign) and in adults are usually cancerous (malignant). The differential diagnosis is done with a metastasis from thyroid carcinoma, cystic squamous cell carcinoma of cervical lymph node, follicular adenomas of the thyroid, lymphangiomas, and bronchial cysts. ${ }^{[6]}$ Complete excision should be done in all cases. The procedure must not be delayed because although 
these lesions are benign which grow quickly. Affected individuals must be monitored periodically to check for any recurrence.

Fine Needle Aspiration cytology can be very helpful for this diagnosis in excluding other differential diagnoses. FNAC a non-invasive procedure saves time and money of the patient. In our case biopsy was not done as the FNAC report was considered conclusive and adviced for complete excision with histopathological correlation. After that patient didn't came to review, so there is no further information whether patient undergone complete excision surgery or not.

\section{Conclusion}

Teratomas are rare benign tumors derived from all three germ cell layers in the cervical region in children. Eventually, compression of vital structures may cause life-threatening complications. FNAC a non-invasive procedure saves time and money of the patient. In our case biopsy was not done as the FNAC report was considered conclusive and adviced for complete excision with histopathological correlation. An early complete surgical approach to congenital cervical teratomas shows good results with low rates of complication and recurrence.

Statement of Consent: Written and informed consent was taken from the parents of this patient for publication of this cases and associated images.

\section{Acknowledgement}

None

\section{Conflict of interest}

None declared

\section{References}

1. Rothschild MA, Catalano P, Urken M, Brandwein M, Som P, Norton K, Biller HF (1994) Evaluation and management of congenital cervical teratoma. Case report and review. Arch Otolaryngol Head Neck Surg 120(4):444-448.

2. Cervical Teratoma - NORD (National Organization for Rare Disorders) [Internet]. NORD (National Organization for Rare Disorders). 2020 [cited 11 December 2020]. Available from: https://rarediseases.org/rare-diseases/cervicalteratoma/

3. Uchiyama M, Iwafuchi $M$, Naitoh $S$, et al. A huge immature cervical teratoma in a newborn: report of a case. Surg Today. $1995 ; 25: 737-740$

4. Miliaras D, Grimbizis G, Conroy J, Psarra N, Miliaras S, Nowak N, et al. Novel karyotypic changes detected by comparative genomic hybridization in a case of congenital cervical immature teratoma. Birth Defects Res A Clin Mol Teratol. 2005;73:572-6.

5. Agarwal G, Kar DK. Teratoma of the anterior mediastinum presenting as a cystic neck mass: a case report. J Med Case Rep. 2008;2:23.

6. Batsakis JG, El-Naggar AK, Luna MA. Teratomas of the head and neck with emphasis on malignancy. Ann Otol Rhinol Laryngol. 1995;104:496-500.

*Corresponding author:

Dr Aarzoo Jahan, North Delhi Municipal Corporation Medical College and Hindu Rao Hospital, Delhi, India

Phone: +91 011-23905652

Email: jahanaarzoo@yahoo.in

Date of Submission : 26/09/2020

Date of Revision $\quad: 11 / 12 / 2020$

Date of Acceptance : 11/12/2020

Date of Publication : 30/01/2021 